\title{
Existence of representation frames based on wave packet groups
}

\author{
Ali Akbar Arefijamaal* (D), Atefe Razghandi (D) \\ Department of Mathematics and Computer Sciences, Hakim Sabzevari University, Sabzevar, Iran
}

\begin{abstract}
Let $H$ be a locally compact group, $K$ a locally compact abelian group with dual group $\widehat{K}$. In this article, we consider the wave packet group $G_{\Theta}$ which is the semidirect product of locally compact groups $H$ and $K \times \widehat{K}$, where $\Theta$ is a continuous homomorphism from $H$ into $A u t(K \times \widehat{K})$. We review the quasi regular representation on $G_{\Theta}$ and extend the continuous Zak transform to $L^{2}\left(G_{\Theta}\right)$. Moreover, we state a continuous frame based on $G_{\Theta}$ to reconstruct the element of $L^{2}(K \times \widehat{K})$. These results are extended to more general wave packet groups. Finally, we establish some methods to find dual of such continuous frames in the form of original frames.
\end{abstract}

Mathematics Subject Classification (2010). 43A30, 42C15

Keywords. semidirect product groups, quasi regular representation, wave packet groups

\section{Introduction and preliminaries}

The concept Zak transform was first introduced and used in 1950 by Gelfand for a problem in differential equations [15]. It was developed by Zak in quantum mechanic [34], and later became a fundamental tool in the analysis of Gabor systems. A basic of the theory and applications to signal analysis can be found in the survey article of [27]. The Zak transform on locally compact abelian groups widely studied by several authors $[3,23,28,30]$. An approach to define the Zak transform on semidirect product groups $G_{\tau}=H \ltimes_{\tau} K$ where $H$ is a locally compact group, $K$ a locally compact abelian group and $\tau: H \rightarrow \operatorname{Aut}(K)$ is a continuous homomorphism can be found in [4]. Many non-abelian groups which appear in mathematical physics and quantum mechanics can be considered as semidirect product of locally compact abelian groups. In this article, we introduce a compatible extension of the Zak transform to such groups, which is a generalization of the Fourier transform. This leads to the development of admissibility conditions on wave packet groups. When $G$ is the Affine group (which is the semidirect product of the two locally compact groups $(\mathbb{R} \backslash\{0\},$.$) and (\mathbb{R},+)$, the group operations on $G$ are

$$
(a, b)(c, d)=(a c, b+a d), \quad(a, b)^{-1}=\left(\frac{1}{a},-\frac{b}{a}\right) .
$$

\footnotetext{
*Corresponding Author.

Email addresses: arefijamaal@hsu.ac.ir (A. Arefijamaal), a.razghandi@hsu.ac.ir (A. Razghandi)

Received: 16.03.2019; Accepted: 11.02.2020
} 
The quasi regular representation $\rho$ of $G$ on the Hilbert space $L^{2}(\mathbb{R})$ is defined by

$$
(\rho(a, b) f)(x)=|a|^{-\frac{1}{2}} f\left(\frac{x-b}{a}\right) .
$$

Then $\rho$ is an irreducible representation of $G$. Moreover, a vector $\psi \in L^{2}(\mathbb{R})$ is an admissible vector for $\rho$ if $C_{\psi}^{2}=\int_{\mathbb{R}} \frac{|\widehat{\psi}(\omega)|^{2}}{|\omega|} d \omega<\infty,[29]$. Many authors have considered, as a general form, the semidirect product group of the locally compact group $H$ and $\mathbb{R}^{n}[4,5,18]$. In [9], Cordoba and Fefferman introduced wave packets by applying collections of dilations, modulations and translations to the Gaussian function. Wave packet transform on $\mathbb{R}$ has been well studied and extended for higher dimension by several authors, see [2,13,16,22]. As other recent direction of wave packet theory see [18-21]. In this paper, we study harmonic analysis properties of quasi regular representation on the wave packet group. Also, we can construct functions of $L^{2}(K \times \widehat{K})$ from the quasi regular coefficients. More precisely, we first establish the representation frames based on a wave packet group; so called the wave packet representation frames on the Hilbert space $L^{2}(K \times \widehat{K})$, then try to characterize their duals. As a well known result; the elements of underlying Hilbert space can be constructed by a pair of dual representation frames. One of our aim is to provide a construction of a pair of dual wave packet frames.

Throughout this article, let $H$ be a locally compact group and $K$ a locally compact abelian group, also let $h \mapsto \tau_{h}$ be a homomorphism of $H$ into the group of automorphisms of $K$ such that the mapping $(h, k) \longmapsto k^{h}:=\tau_{h}(k)$ from $H \times K$ onto $\mathrm{K}$ is continuous. Then the set $H \times K$ with the product topology and the operations

$$
\begin{aligned}
(h, k) \cdot\left(h^{\prime}, k^{\prime}\right) & =\left(h h^{\prime}, k \tau_{h}\left(k^{\prime}\right)\right), \quad\left((h, k) \in G_{\tau}\right) \\
(h, k)^{-1} & =\left(h^{-1}, \tau_{h^{-1}}\left(k^{-1}\right)\right)
\end{aligned}
$$

which is a (not necessarily abelian) locally compact group, is called the semidirect product of $H$ and $K$, respectively. This group is denoted by $G_{\tau}=H \ltimes_{\tau} K$ and its left Haar measure is given by $d m_{G_{\tau}}(h, k)=\delta(h) d m_{H}(h) d m_{K}(k)$ where $d m_{H}(h)$ and $d m_{K}(k)$ are the left Haar measures of $H$ and $K$ respectively, and the positive continuous homomorphism $\delta$ on $H$ is given by (15.29 of [26])

$$
d m_{K}(k)=\delta(h) d m_{K}\left(\tau_{h}(k)\right) .
$$

The above $\tau$-dual action on $K$ induces the homomorphism $\widehat{\tau}: H \rightarrow A u t(\widehat{K})$ via $h \mapsto \widehat{\tau}_{h}$, given by

$$
\widehat{\tau}_{h}(\omega):=\omega_{h}=\omega \circ \tau_{h^{-1}}
$$

for all $\omega \in \widehat{K}$, where $\omega_{h}(k)=\omega\left(\tau_{h^{-1}}(k)\right)$ for all $k \in K$ [4]. Also, $(h, \omega) \mapsto \omega \circ \tau_{h}$ from $H \times \widehat{K}$ into $\widehat{K}$ is continuous. Hence, the semidirect product $G_{\widehat{\tau}}=H \times_{\widehat{\tau}} \widehat{K}$, which is a locally compact group, can play the role of dual for $G_{\tau}$. Furthermore, the continuous action $(h, k) \mapsto \tau_{h}(k)$ induces a mapping $\Theta: H \rightarrow A u t(K \times \widehat{K})$ given by $h \mapsto \Theta_{h}$ where

$$
\Theta_{h}(k, \omega)=\left(\tau_{h}(k), \omega_{h}\right) .
$$

In [20], it is shown that $\Theta$ is well defined and $(h, k, \omega) \mapsto \Theta_{h}(k, \omega)$ is a continuous homomorphism. Thus $\Theta$ induces the semidirect product group $G_{\Theta}=H \ltimes_{\Theta}(K \times \widehat{K})$ which is called the wave packet group. It is a locally compact group with the left Haar measure

$$
d m_{\Theta}(h, k, \omega)=d m_{H}(h) d m_{K}(k) d m_{\widehat{K}}(\omega)
$$

and the modular function

$$
\Delta_{G_{\Theta}}(h, k, \omega)=\Delta_{H}(h),
$$

for all $(h, k, \omega) \in G_{\Theta}$, for more details see Theorem 3.2 of [20]. 
Suppose $G$ is a locally compact abelian group with the left Haar measure $m_{G}$, the Fourier transform $\widehat{f}$ of any function $f \in L^{1}(G)$, denoted by $\mathcal{F}(f)$ or $\widehat{f}$, is defined by

$$
\widehat{f}(\xi)=\int_{G} f(x) \overline{\xi(x)} d m_{G}(x) .
$$

The Fourier transform can be extended to an isometry from $L^{2}(G)$ onto $L^{2}(\widehat{G})$, the socalled Plancherel isomorphism. By the Fourier inversion, we can reconstruct a function from its Fourier transform as following

$$
f(x)=\int_{\widehat{G}} \widehat{f}(\xi) \xi(x) d m_{\widehat{G}}(\xi)
$$

for all $f \in L^{1}(G)$ and $\widehat{f} \in L^{1}(\widehat{G})$. For $(k, \omega) \in K \times \widehat{K}$ the translation operator $T_{(k, \omega)}$ is defined on $L^{2}(K \times \widehat{K})$ by

$$
T_{(k, \omega)} f(x, \xi)=f\left(x k^{-1}, \xi \bar{\omega}\right) .
$$

Also the modulation of $f$ by $(\omega, x)$ is defined as

$$
M_{(\omega, x)} f(y, \xi)=\overline{\omega(y) \xi(x)} f(y, \xi), \quad((\omega, x) \in \widehat{K} \times K) .
$$

The Fourier transform has served as a bridge between unitary operators modulation and translation as

$$
\widehat{M_{(\omega, k)}} f=T_{\left(\bar{\omega}, k^{-1}\right)} \widehat{f}, \quad \widehat{T_{(k, \omega)} f}=M_{(k, \omega)} \widehat{f},
$$

for all $f \in L^{2}(K \times \widehat{K}), k \in K$ and $\omega \in \widehat{K}$.

Let $(\pi, \mathcal{H})$ be a unitary representation of $\mathrm{G}$. By an admissible vector we mean a vector $\psi \in \mathcal{H}$ such that

$$
\int_{G}|\langle f, \pi(x) \psi\rangle|^{2} d m_{G}(x)<\infty, \quad(f \in \mathcal{H}) .
$$

If $\pi$ is irreducible (1.3) is equivalent to

$$
C_{\psi}:=\int_{G}|\langle\psi, \pi(x) \psi\rangle|^{2} d m_{G}(x)<\infty .
$$

Also we have the reconstruction formula as

$$
f=C_{\psi}^{-1 / 2} \int_{G}\langle f, \pi(x) \psi\rangle \pi(x) \psi d m_{G}(x), \quad(f \in \mathcal{H}),
$$

for more details see [1].

For every semidirect product $G_{\tau}=H \ltimes_{\tau} K$, the quasi regular representation $\left(U_{\tau}, L^{2}(K)\right)$ on $G_{\tau}$ is defined by

$$
\left(U_{\tau}(h, k) f\right)(x)=\delta(h)^{-1 / 2} f\left(\tau_{h^{-1}}\left(x k^{-1}\right)\right), \quad\left(f \in L^{2}(K)\right) .
$$

It is not irreducible, in general, see [5] for more details.

Let $\mathcal{H}$ be a separable Hilbert space and $X$ a locally compact Hausdorff space endowed with a positive Radon measure $\nu$. A mapping $F: X \rightarrow \mathcal{H}$ is called a continuous frame if the mapping $x \rightarrow\langle F(x), f\rangle$ is measurable for all $f \in \mathcal{H}$ and there exist constants $0<A, B<+\infty$ such that

$$
A\|f\|^{2} \leq \int_{X}|\langle F(x), f\rangle|^{2} d \nu(x) \leq B\|f\|^{2} \quad(f \in \mathcal{H}) .
$$

A continuous frame is said to be tight when $A=B$. The mapping $F$ is called Bessel if the second inequality in (1.6) holds. Suppose that $F$ is Bessel, then the operator $T_{F}$ : $L^{2}(X) \rightarrow \mathcal{H}$ defined weakly by

$$
T_{F} u=\int_{X} u(x) F(x) d \nu(x)
$$


is a bounded linear operator; so called the synthesis operator. The continuous frame operator is defined to be $S_{F}=T_{F} T_{F}^{*}$ [7]. It is useful to reconstruct the elements of $\mathcal{H}$ as

$$
f=S_{F}^{-1} S_{F} f=\int_{X}\langle f, F(x)\rangle S_{F}^{-1} F(x) d \nu(x) .
$$

The paper is organized as follows. In Section 2, we introduce the concept of Zak transform on wave packet group $G_{\Theta}$ and give sufficient admissibility condition for the quasi regular representation on $G_{\Theta}$. This leads to obtain the reconstruction formula and orthogonality relation. Section 3 is devoted to frame analysis on generalized wave packet groups. In Section 4, we establish a method to find dual of representation frames on wave packet and generalized wave packet groups. Finally, in Section 5, we give some examples to illustrate our results.

\section{Continuous Zak transforms on abstract wave packet groups}

Throughout this paper, we assume that $\mathrm{H}$ is a locally compact group with the left Haar measure $m_{H}$ and $K$ a locally compact abelian group with the dual group $\widehat{K}$. We also denote the normalized Plancherel measure of $\widehat{K}$ by $m_{\widehat{K}}$. In this section, we state a representation frame based on $G_{\Theta}$, where the homomorphism $\Theta$ is defined by (1.1). It is worthwide to mention that the dilation operator and its Fourier transform, denoted respectively by $D_{h}$ and $\widehat{D}_{h}$ for every $h \in H$, are defined by [20]

$$
\begin{array}{cc}
D_{h} f(k)=\delta(h)^{1 / 2} f\left(\tau_{h^{-1}}(k)\right) & \left(f \in L^{2}(K)\right), \\
\widehat{D}_{h} g(\omega)=\delta(h)^{-1 / 2} g\left(\widehat{\tau}_{h^{-1}}(\omega)\right) & \left(g \in L^{2}(\widehat{K})\right) .
\end{array}
$$

Moreover, the dilation operator extends on $L^{2}(K \times \widehat{K})$ by

$$
\mathfrak{D}_{h} \phi(k, \omega)=\phi\left(\tau_{h^{-1}}(k), \widehat{\tau}_{h^{-1}}(\omega)\right),
$$

and

$$
\widehat{\mathfrak{D}}_{h} \psi(\omega, k)=\psi\left(\widehat{\tau}_{h^{-1}}(\omega), \tau_{h^{-1}}(k)\right),
$$

where $\psi \in L^{2}(\widehat{K} \times K)$ and $\phi \in L^{2}(K \times \widehat{K})$. We summerize the basic properties of the dilation operators $\mathfrak{D}_{h}$ and $\widehat{\mathfrak{D}_{h}}$ on wave packet group $G_{\Theta}$ in the following:

Lemma 2.1. For every wave packet group $G_{\Theta}=H \ltimes_{\Theta}(K \times \widehat{K})$ the following assertions hold.

(1) The mapping $h \mapsto \mathfrak{D}(h):=\mathfrak{D}_{h}$ defines a continuous unitary representation of $H$ on the Hilbert space $L^{2}(K \times \widehat{K})$.

(2) The maping $h \mapsto \widehat{\mathfrak{D}}(h):=\widehat{\mathfrak{D}}_{h}$ defines a continuous unitary representation of $H$ on the Hilbert space $L^{2}(\widehat{K} \times K)$.

(3) $\widehat{\mathfrak{D}_{h} f}=\widehat{\mathfrak{D}_{h}} \widehat{f}$, for all $h \in H$ and $f \in L^{2}(K \times \widehat{K})$.

(4) $\mathfrak{D}_{h} T_{(k, \omega)}=T_{\left(k^{h}, \omega_{h}\right)} \mathfrak{D}_{h}$.

(5) $T_{(k, \xi)} M_{(\omega, x)}=\omega(k) \xi(x) M_{(\omega, x)} T_{(k, \xi)}$.

(6) $\mathfrak{D}_{h} M_{(\omega, x)}=M_{\left(\omega_{h}, x^{h}\right)} \mathfrak{D}_{h}$. 
Proof. (1) If $f \in L^{2}(K \times \widehat{K})$ then for $h \in H, k \in K$ and $\omega \in \widehat{K}$ we have

$$
\begin{aligned}
\left\|\mathfrak{D}_{h} \psi\right\|_{L^{2}(K \times \widehat{K})}^{2} & =\int_{K} \int_{\widehat{K}}\left|\mathfrak{D}_{h} \psi(k, \omega)\right|^{2} d m_{K}(k) d m_{\widehat{K}}(\omega) \\
& =\int_{K} \int_{\widehat{K}}\left|\psi\left(\tau_{h^{-1}}(k), \widehat{\tau}_{h^{-1}}(\omega)\right)\right|^{2} d m_{K}(k) d m_{\widehat{K}}(\omega) \\
& =\int_{K} \int_{\widehat{K}}|\psi(k, \omega)|^{2} d m_{K}\left(\tau_{h}(k)\right) d m_{\widehat{K}}\left(\widehat{\tau}_{h}(\omega)\right) \\
& =\int_{K} \int_{\widehat{K}}|\psi(k, \omega)|^{2} d m_{K}(k) d m_{\widehat{K}}(\omega) \\
& =\|\psi\|_{L^{2}(K \times \widehat{K})}^{2}
\end{aligned}
$$

Thus $\mathfrak{D}_{h}: L^{2}(K \times \widehat{K}) \rightarrow L^{2}(K \times \widehat{K})$ is an isometry. Also $\mathfrak{D}_{h \hat{h}}=\mathfrak{D}_{h} \mathfrak{D}_{\hat{h}}$ for $h, h^{\prime} \in H$. So the map $\mathfrak{D}: H \rightarrow \mathcal{U}\left(L^{2}(\widehat{K} \times K)\right)$ given by $h \mapsto \mathfrak{D}(h):=\mathfrak{D}_{h}$ is a continuous unitary representation of $H$ on the Hilbert space $L^{2}(\widehat{K} \times K)$.

The rest follows immediately.

The quasi regular representation of $G_{\Theta}$ can be stated by the quasi regular representations of $G_{\tau}$ and $G_{\widehat{\tau}}$.

Theorem 2.2. Let $\left(U_{\tau}, L^{2}(K)\right)$ and $\left(U_{\widehat{\tau}}, L^{2}(\widehat{K})\right)$ be the quasi regular representations on $G_{\tau}$ and $G_{\widehat{\tau}}$ respectively. Then

(1) $G_{\Theta}$ is isomorphic with the subgroup $D:=\{(h, k, h, \omega): h \in H, k \in K, \omega \in \widehat{K}\}$ of $G_{\tau} \times G_{\widehat{\tau}}$.

(2) The restriction of the representation $U_{\tau} \otimes U_{\widehat{\tau}}$ to the subgroup $D$ is unitary equivalent to the quasi regular representation on wave packet group $G_{\Theta}$.

Proof. (1) Define $\Phi: G_{\Theta} \rightarrow G_{\tau} \times G_{\widehat{\tau}}$ by $\Phi(h, k, \omega)=((h, k),(h, \omega))$. Clearly, it is an one to one homomorphis. This easily follows (1). In order to show (2) notice that the quasi regular representation $U$ on $G_{\Theta}$ acts on $L^{2}(K \times \widehat{K})$ by

$$
U(h, k, \omega) \varphi(x, \xi)=T_{(k, \omega)} \mathfrak{D}_{h} \varphi(x, \xi) .
$$

Define the operator $\Gamma: L^{2}(K) \otimes L^{2}(\widehat{K}) \rightarrow L^{2}(K \times \widehat{K})$, by $f_{1} \otimes f_{2} \mapsto f_{1} \times f_{2}$. Due to Section 5.3 of [33], it is enough to show that $\Gamma$ is an intertwing operator only on the tensor product elements $f_{1} \otimes f_{2}$. In fact,

$$
\begin{aligned}
U(h, k, \omega) \Gamma\left(f_{1} \otimes f_{2}\right)(x, \xi) & =U(h, k, \omega)\left(f_{1} \times f_{2}\right)(x, \xi) \\
& =f_{1}\left(\tau_{h^{-1}}\left(x k^{-1}\right)\right) f_{2}\left((\xi \bar{\omega})_{h^{-1}}\right) \\
& =\Gamma\left(U_{\tau}(h, k) f_{1}(x) \otimes U_{\widehat{\tau}}(h, \omega) f_{2}(\xi)\right) \\
& =\Gamma\left(U_{\tau} \otimes U_{\widehat{\tau}}\right)((h, k)(h, \omega)) f_{1} \otimes f_{2}(x, \xi) .
\end{aligned}
$$

Now we try to extend the notion of Zak transform to wave packet groups. The continuous Zak transform of $F \in C_{c}\left(G_{\Theta}\right)$ is defined on $G_{\Theta}$ by

$$
Z_{c} F(h, \omega, x)=\int_{K} \int_{\widehat{K}} F(h, y, \gamma) \overline{\omega(y) \gamma(x)} d m_{K}(y) d m_{\widehat{K}}(\gamma)
$$


Suppose $F=f \otimes \varphi$ where $f \in C_{c}(H)$ and $\varphi \in C_{c}(K \times \widehat{K})$ then $F$ can be considered as an element of $L^{2}\left(G_{\Theta}\right)$. Using the inversion formula (1.2) we obtain

$$
\begin{aligned}
& \left\|Z_{c} F\right\|_{2}^{2}=\int_{G_{\Theta}}\left|\int_{K} \int_{\widehat{K}} f(h) \varphi(y, \gamma) \overline{\omega(y) \gamma(x)} d m_{K}(y) d m_{\widehat{K}}(\gamma)\right|^{2} d m_{H}(h) d m_{\widehat{K}}(\omega) d m_{K}(x) \\
& \quad=\int_{H}|f(h)|^{2} d m_{H}(h) \int_{K} \int_{\widehat{K}}\left|\int_{K} \int_{\widehat{K}} \varphi(y, \gamma) \overline{\omega(y) \gamma(x)} d m_{K}(y) d m_{\widehat{K}}(\gamma)\right|^{2} d m_{\widehat{K}}(\omega) d m_{K}(x) \\
& \quad=\|f\|_{2}^{2}\|\widehat{\varphi}\|_{2}^{2} \\
& \quad=\|f \otimes \varphi\|_{2}=\|F\|_{2} .
\end{aligned}
$$

So, $Z_{c}$ is a linear isometry in $L^{2}-$ norm on $C_{c}\left(G_{\Theta}\right)$ and therefore can be extended uniquely to the continuous Zak transform $Z_{c}: L^{2}\left(G_{\Theta}\right) \rightarrow L^{2}\left(G_{\widehat{\Theta}}\right)$. In particular $Z_{c}(f \otimes \varphi)=f \otimes \widehat{\varphi}$, for every $f \in L^{2}(H)$ and $\varphi \in L^{2}(K \times \widehat{K})$. Hence, $Z_{c}$ on $L^{2}(K \times \widehat{K})$ is the same classical Fourier transform.

In the next, we need the following lemma to state our main result.

Lemma 2.3. Let $\psi \in L^{2}(K \times \widehat{K})$. Then

$$
Z_{c} T_{(k, \omega)} \mathfrak{D}_{h} \psi(\eta, x)=\overline{\eta(k) \omega(x)} Z_{c} \mathfrak{D}_{h} \psi(\eta, x), \quad((\eta, x) \in \widehat{K} \times K)
$$

where $h \in H, k \in K$ and $\omega \in \widehat{K}$.

Proof. Using (2.1), for every $\psi \in L^{2}(K \times \widehat{K})$ we obtain

$$
\begin{aligned}
Z_{c} T_{(k, \omega)} \mathfrak{D}_{h} \psi(\eta, x) & =\int_{K} \int_{\widehat{K}} D_{h} \psi\left(y k^{-1}, \gamma \bar{\omega}\right) \overline{\eta(y) \gamma(x)} d m_{K}(y) d m_{\widehat{K}}(\gamma) \\
& =\int_{K} \int_{\widehat{K}} D_{h} \psi(y, \gamma) \overline{\eta(y k) \gamma \omega(x)} d m_{K}(y) d m_{\widehat{K}}(\gamma) \\
& =\overline{\eta(k) \omega(x)} \int_{K} \int_{\widehat{K}} D_{h} \psi(y, \gamma) \overline{\eta(y) \gamma(x)} d m_{K}(y) d m_{\widehat{K}}(\gamma) \\
& =\overline{\eta(k) \omega(x)} Z_{c} \mathfrak{D}_{h} \psi(\eta, x),
\end{aligned}
$$

for every $h \in H, k \in K$ and $\omega \in \widehat{K}$.

Theorem 2.4. Let $\left(U, L^{2}(K \times \widehat{K})\right)$ be the quasi regular representation on the wave packet group $G_{\Theta}=H \ltimes_{\Theta}(K \times \widehat{K})$ and $\psi \in L^{2}(K \times \widehat{K})$. Then

(1) The family $\mathbb{F}(\psi):=\left\{T_{(k, \omega)} \mathfrak{D}_{h} \psi ; \quad(h, k, \omega) \in G_{\Theta}\right\}$ is a representation frame based on $G_{\Theta}$ with bounds $A$ and $B$ if and only if $A \leq \gamma_{\psi} \leq B$ a.e., where

$$
\gamma_{\psi}(\xi, y):=\int_{H}\left|\left(Z_{c} \mathfrak{D}_{h} \psi\right)(\xi, y)\right|^{2} d m_{H}(h)
$$

(2) $\psi$ is an admissible vector if and only if $\left\|\gamma_{\psi}\right\|_{\infty}<\infty$.

Proof. (1) Let $\mathbb{F}(\psi)$ be a continuous frame with bounds $A, B$ and $\varphi \in L^{2}(K \times \widehat{K})$. Then the frame operator of $\mathbb{F}(\psi)$ on $\varphi$, which is given by

$$
S_{\psi} \varphi=\int_{G_{\Theta}}\left\langle\varphi, T_{(k, \omega)} \mathfrak{D}_{h} \psi\right\rangle T_{(k, \omega)} \mathfrak{D}_{h} \psi d m_{H}(h) d m_{K}(k) d m_{\widehat{K}}(\omega),
$$


defines as element of $L^{2}(K \times \widehat{K})$ weakly. Using Lemma 2.3 and inversion formula (1.2) yields

$$
\begin{aligned}
\left(Z_{c} S_{\psi} \varphi\right)(\xi, y) & =\int_{G_{\Theta}}\left\langle\varphi, T_{(k, \omega)} \mathfrak{D}_{h} \psi\right\rangle Z_{c} T_{(k, \omega)} \mathfrak{D}_{h} \psi(\xi, y) d m_{H}(h) d m_{K}(k) d m_{\widehat{K}}(\omega) \\
& =\frac{\int_{H}\left(Z_{c} \mathfrak{D}_{h} \psi\right)(\xi, y) \int_{K} \int_{\widehat{K}} \int_{K} \int_{\widehat{K}}\left(Z_{c} \varphi \overline{Z_{c} \mathfrak{D}_{h} \psi}\right)(\gamma, s) \gamma(k) \omega(s) \overline{\xi(k)}}{\omega(y)} d m_{H}(h) d m_{\widehat{K}}(\gamma) d m_{K}(s) d m_{\widehat{K}}(\omega) d m_{K}(k) \\
& =\int_{H}\left(Z_{c} \mathfrak{D}_{h} \psi\right)(\xi, y) Z_{c} \varphi(\xi, y) \overline{\left(Z_{c} \mathfrak{D}_{h} \psi\right)(\xi, y)} d m_{H}(h) \\
& =Z_{c} \varphi(\xi, y) \gamma_{\psi}(\xi, y)
\end{aligned}
$$

where $\gamma_{\psi}$, as a function on $K \times \widehat{K}$, is given by (2.2). In particular,

$$
\widehat{S_{\psi} \varphi}=\gamma_{\psi} \widehat{\varphi} \text {. }
$$

Thus,

$$
A\|\varphi\|_{2}^{2} \leq\left\langle Z_{c} \varphi \gamma_{\psi}, Z_{c} \varphi\right\rangle \leq B\|\varphi\|_{2}^{2} . \quad\left(\varphi \in L^{2}(K \times \widehat{K})\right),
$$

or equivalently,

$$
A \leq \gamma_{\psi} \leq B \text {, a.e.. }
$$

Conversely, assume that $A \leq \gamma_{\psi} \leq B$ a.e., using the above computations we obtain

$$
\begin{aligned}
& \left\langle\int_{G_{\Theta}}\left\langle\varphi, T_{(k, \omega)} \mathfrak{D}_{h} \psi\right\rangle T_{(k, \omega)} \mathfrak{D}_{h} \psi d m_{H}(h) d m_{K}(k) d m_{\widehat{K}}(\omega), \varphi\right\rangle \\
= & \left\langle\int_{G_{\Theta}}\left\langle\varphi, T_{(k, \omega)} \mathfrak{D}_{h} \psi\right\rangle Z_{c} T_{(k, \omega)} \mathfrak{D}_{h} \psi d m_{H}(h) d m_{K}(k) d m_{\widehat{K}}(\omega), Z_{c} \varphi\right\rangle \\
= & \left\langle\gamma_{\psi} \widehat{\varphi}, \widehat{\varphi}\right\rangle .
\end{aligned}
$$

i.e. $\mathbb{F}(\psi)$ is a frame with bounds $A$ and $B$.

(2) Suppose $\left\|\gamma_{\psi}\right\|_{\infty} \leq \infty$, then $\mathbb{F}(\psi)$ is a Bessel sequence. Applying (2.3) we have

$$
\begin{aligned}
C_{\psi} & :=\int_{G_{\Theta}}\left|\left\langle\psi, T_{(k, \omega)} \mathcal{D}_{h} \psi\right\rangle\right|^{2} d m_{\widehat{K}}(\omega) d m_{K}(k) d m_{H}(h) \\
& =\left\langle\widehat{S_{\psi} \psi}, \widehat{\psi}\right\rangle=\left\langle\gamma_{\psi} \widehat{\psi}, \widehat{\psi}\right\rangle
\end{aligned}
$$

So $C_{\psi} \leq\left\|\gamma_{\psi}\right\|_{\infty}\|\psi\|_{2}<\infty$. The converse is follows immediately.

The representation frame $\mathbb{F}(\psi)$, introduced in the above theorem, is called the wave packet representation frame associated to $\psi \in L^{2}(K \times \widehat{K})$.

Corollary 2.5. Let $G_{\Theta}=H \ltimes_{\Theta}(K \times \widehat{K})$ and $\psi \in L^{2}(K \times \widehat{K})$ generates wave packet representation frame $\mathbb{F}(\psi)$. Then

(1) The following reconstruction formula for the elements of $L^{2}(K \times \widehat{K})$ holds

$$
\varphi=\int_{G_{\Theta}}\left\langle\widehat{\varphi}, \gamma_{\psi}^{-1} M_{(k, \omega)} \widehat{\mathfrak{D}_{h}} \widehat{\psi}\right\rangle T_{(k, \omega)} \mathfrak{D}_{h} \psi d m_{H}(h) d m_{\widehat{K}}(\omega) d m_{K}(k)
$$

(2) The optimal frame bounds of $\mathbb{F}(\psi)$ are given by $\left\|\gamma_{\psi}^{-1}\right\|_{\infty}^{-1}$ and $\left\|\gamma_{\psi}\right\|_{\infty}$, respectively. 
Proof. (1) Using Theorem 2.4 follows that

$$
\left\langle\widehat{S_{\psi} \varphi}, \widehat{\varphi}\right\rangle=\left\langle\gamma_{\psi} \widehat{\varphi}, \widehat{\varphi}\right\rangle, \quad\left(\varphi \in L^{2}(K \times \widehat{K})\right)
$$

where $S_{\psi}$ is the frame operator of $\mathbb{F}(\psi)$. This easily follows that

$$
\left.\widehat{\left(S_{\psi}^{-1} \varphi\right.}\right)=\gamma_{\psi}^{-1} \widehat{\varphi}
$$

Therefore, we can rewrite (1.7) as

$$
\begin{aligned}
\varphi & =\int_{G_{\Theta}}\left\langle\widehat{\varphi}, \mathcal{F}\left(S^{-1} T_{(k, \omega)} \mathfrak{D}_{h} \psi\right)\right\rangle T_{(k, \omega)} \mathfrak{D}_{h} \psi d m_{H}(h) d m_{\widehat{K}}(\omega) d m_{K}(k) \\
& =\int_{G_{\Theta}}\left\langle\widehat{\varphi}, \gamma_{\psi}{ }^{-1} M_{(\omega, k)} \widehat{\mathfrak{D}_{h}} \widehat{\psi}\right\rangle T_{(k, \omega)} \mathfrak{D}_{h} \psi d m_{H}(h) d m_{\widehat{K}}(\omega) d m_{K}(k) .
\end{aligned}
$$

(2) Applying (2.3) yields $\left\|\widehat{S_{\psi}}\right\|=\left\|\gamma_{\psi}\right\|_{\infty}$. Hence

$$
\left\|S_{\psi}\right\|=\left\|\gamma_{\psi}\right\|_{\infty} \text {. }
$$

Using Proposition 5.1.1 of [24] follows the desired results.

As usual, by using the quasi regular representation $U$ on $G_{\Theta}$, for an admissible vector $\psi \in L^{2}(K \times \widehat{K})$ one can define the wave packet transform on $L^{2}(K \times \widehat{K})$ by

$$
W_{\psi} \varphi(h, k, \omega):=\langle\varphi, U(h, k, \omega) \psi\rangle=\left\langle\varphi, T_{(k, \omega)} \mathfrak{D}_{h} \psi\right\rangle .
$$

In this case, the reconstruction formula (1.7) can be read as

$$
\varphi=C_{\psi}{ }^{-1 / 2} \int_{G_{\Theta}}\left\langle\varphi, T_{(k, \omega)} \mathcal{D}_{h} \psi\right\rangle T_{(k, \omega)} \mathcal{D}_{h} \psi d m_{H}(h) d m_{K}(k) d m_{\widehat{K}}(\omega),
$$

which is different from the inversion formula (2.4). In fact, (2.6) is followed from the continuous wavelet theory and is obtained by the constant $C_{\psi}$, see also (1.4). However, (2.4) is the reconstruction formula (1.6) and follows from the frame theory.

Corollary 2.6. Let $\mathbb{F}(\psi)$ be the representation frame given by Theorem 2.4. Then the wave packet transform satisfies the following orthogonality relation

$$
\left\langle W_{\psi} \varphi, W_{\psi} \eta\right\rangle=\left\langle\gamma_{\psi} \widehat{\varphi}, \widehat{\eta}\right\rangle .
$$

As a consequence of Theorem 2.4, we can construct the admissible vectors of $\left(U, L^{2}(K \times \widehat{K})\right)$ by using its components.

Corollary 2.7. Let $\psi_{1} \in L^{2}(K)$ and $\psi_{2} \in L^{2}(\widehat{K})$. The following assertions hold.

(1) If $\psi_{1}$ is an admissible vector with respect to the representation $\left(U_{\tau}, L^{2}(K)\right)$ and $\psi_{2} \in L^{2}(\widehat{K})$ is bounded, then $\psi_{1} \otimes \psi_{2}$ is admissible with respect to the representation $\left(U, L^{2}(K \times \widehat{K})\right)$.

(2) If $\psi_{2}$ is an admissible vector with respect to the representation $\left(U_{\widehat{\tau}}, L^{2}(\widehat{K})\right)$ and $\psi_{1} \in L^{2}(K)$ is bounded, then $\psi_{1} \otimes \psi_{2}$ is admissible with respect to the representation $\left(U, L^{2}(K \times \widehat{K})\right)$.

(3) The vector $\psi \in L^{2}(K \times \widehat{K})$ defined as

$$
|\widehat{\psi}|=\left|\widehat{\psi}_{1}\right|^{1 / 2} \otimes\left|\widehat{\psi}_{2}\right|^{1 / 2}
$$

is an admissible vector for $\left(U, L^{2}(K \times \widehat{K})\right)$ provided that $\psi_{1}$ and $\psi_{2}$ are admissible. 
Proof. (1) Putting $\Psi=\psi_{1} \otimes \psi_{2} \in L^{2}(K \times \widehat{K})$. Using the continuous Zak transform we obtain

$$
\begin{aligned}
\gamma_{\Psi}(\omega, x) & =\int_{H}\left|Z_{c} \mathfrak{D}_{h} \Psi(\omega, x)\right|^{2} d m_{H}(h) \\
& =\int_{H}\left|Z_{c}\left(D_{h} \psi_{1} \otimes \widehat{D}_{h} \psi_{2}\right)(\omega, x)\right|^{2} d m_{H}(h) \\
& =\int_{H}\left|\int_{K} D_{h} \psi_{1}(y) \overline{\omega(y)} d m_{K}(y) \int_{\widehat{K}} \widehat{D}_{h} \psi_{2}(\gamma) \overline{\gamma(x)} d m_{\widehat{K}}(\gamma)\right|^{2} d m_{H}(h) \\
& =\int_{H}\left|\left(Z_{c} D_{h} \psi_{1} \otimes Z_{c} \widehat{D}_{h} \psi_{2}\right)(\omega, x)\right|^{2} d m_{H}(h) \\
& \leq \int_{H}\left|\widehat{\psi}_{1}\left(w \circ \tau_{h}\right)\right|^{2} d m_{H}(h)\left\|\widehat{\psi}_{2}\right\|_{\infty}<\infty .
\end{aligned}
$$

The result follows from Theorem 2.4.

(2) For $(\omega, x) \in \widehat{K} \rtimes K$ we have

$$
\begin{aligned}
\gamma_{\psi}(\omega, x) & =\int_{H}\left|Z_{c} \mathfrak{D}_{h} \psi(\omega, x)\right|^{2} d m_{H}(h) \\
& =\int_{H}\left|\widehat{\psi}\left(\widehat{\tau}_{h^{-1}}(\omega), \tau_{h^{-1}}(x)\right)\right|^{2} d m_{H}(h) \\
& =\int_{H}\left|\widehat{\psi}_{1}\left(\widehat{\tau}_{h^{-1}}(\omega)\right)\right| \otimes\left|\widehat{\psi}_{2}\left(\tau_{h^{-1}}(x)\right)\right| d m_{H}(h) \\
& \leq \gamma_{\psi_{1}}(\omega)^{1 / 2} \gamma_{\psi_{2}}(x)^{1 / 2}
\end{aligned}
$$

it follows the results.

\section{Frame analysis on generalized wave packet groups}

Gabor systems generated by the action of translations and modulations on a signal give the time- frequency content of a signal. This is often not the most desired resolution, which leads to the appearance of wavelet analysis. Wavelet systems are obtained by shifting and dilating a finite family of functions. In [9], Cordoba and Fefferman by using certain collections of dilations, modulations and translations, introduced wave packet systems. In fact, Gabor systems and wavelet systems can be considered as special cases of wave packet systems. In this section, we show that the interplay of all three operators gives generalized wave packet system on locally compact groups, this leads up to sufficient conditions that generalized wave packet system be the tight frame.

Let $H$ be a locally compact group and $K$ be a locally compact abelian group. The action $H \times K \rightarrow K ;(h, k) \mapsto \tau_{h}(k)$ and its dual indicate us to consider the action

$$
\begin{aligned}
H \times(K \times \widehat{K} \times \widehat{K} \times K) & \rightarrow K \times \widehat{K} \times \widehat{K} \times K \\
(h, k, \omega, \xi, x) & \mapsto \wp_{h}(k, \omega, \xi, x):=\left(k^{h}, \omega_{h}, \xi_{h}, x^{h}\right) .
\end{aligned}
$$

It is not difficult to see that $\wp_{h}$ is a homomorphism on $K \times \widehat{K} \times \widehat{K} \times K$, and therefore, the semidirect product $G_{\wp}:=H \ltimes_{\wp}(K \times \widehat{K} \times \widehat{K} \times K)$; so called the generalized wave packet group, is well defined. A direct calculations shows that

$$
\begin{aligned}
d m_{G_{\wp}}(h, k, \omega, \xi, x) & =d m_{H}(h) d m_{K}(k) d m_{\widehat{K}}(\omega) d m_{\widehat{K}}(\xi) d m_{K}(x) \\
\triangle_{G_{\wp}}(h, k, \omega, \xi, x) & =\triangle_{H}(h) .
\end{aligned}
$$

Let $\psi \in L^{2}(K \times \widehat{K})$. The generalized wave packet system

$$
\alpha(\psi):=\left\{\mathfrak{D}_{h} T_{(k, \omega)} M_{(\xi, x)} \psi ; \quad(h, k, \omega, \xi, x) \in G_{\wp}\right\}
$$


introduces the unitary representation $\left(\Lambda, L^{2}(K \times \widehat{K})\right)$ on $G_{\wp}$ given by

$$
\Lambda(h, k, \omega, \xi, x) \psi:=\mathfrak{D}_{h} T_{(k, \omega)} M_{(\xi, x)} \psi .
$$

In the rest, we discuss on the irreducibility of $\Lambda$ and characterize its admissible vector.

Theorem 3.1. The homomorphism $\left(\Lambda, L^{2}(K \times \widehat{K})\right)$ on the generalized wave packet group $G_{\wp}$ is a continuous unitary irreducible representation.

Proof. Obviously, $\Lambda$ is a continuous unitary representation. In order to show the irreducibility of $\Lambda$, let $M$ be a nonzero closed invariant subspace of $L^{2}(K \times \widehat{K})$. Then we claim that $M=L^{2}(K \times \widehat{K})$, or equivalently $M^{\perp}=\{0\}$. Let $\varphi \in M^{\perp}$ and $\psi \in M$ be nonzero. Then for each $h_{0} \in H$ we have

$$
\begin{aligned}
0 & =\int_{K} \int_{\widehat{K}} \int_{K} \int_{\widehat{K}}\left|\left\langle\Lambda\left(h_{0}, k, \omega, \xi, x\right) \psi, \varphi\right\rangle\right|^{2} d m_{K}(k) d m_{\widehat{K}}(\omega) d m_{\widehat{K}}(\xi) d m_{K}(x) \\
& =\int_{K} \int_{\widehat{K}} \int_{K} \int_{\widehat{K}}\left|\left\langle D_{h_{0}} T_{(k, \omega)} M_{(\xi, x)} \psi, \varphi\right\rangle\right|^{2} d m_{K}(k) d m_{\widehat{K}}(\omega) d m_{\widehat{K}}(\xi) d m_{K}(x) \\
& =\int_{K} \int_{\widehat{K}} \int_{K} \int_{\widehat{K}}\left|\left\langle M_{(\xi, x)} \psi, T_{\left(k^{-1}, \bar{\omega}\right)} D_{h_{0}^{-1}} \varphi\right\rangle\right|^{2} d m_{K}(k) d m_{\widehat{K}}(\omega) d m_{\widehat{K}}(\xi) d m_{K}(x) \\
& =\int_{K} \int_{\widehat{K}} \int_{K} \int_{\widehat{K}}\left|\mathcal{F}\left(\psi \cdot \overline{T_{\left(k^{-1}, \bar{\omega}\right)} D_{h_{0}^{-1}} \varphi}\right)(\xi, x)\right|^{2} d m_{K}(k) d m_{\widehat{K}}(\omega) d m_{\widehat{K}}(\xi) d m_{K}(x) \\
& =\int_{K} \int_{\widehat{K}}|\psi(\xi, x)|^{2} d m_{\widehat{K}}(\xi) d m_{K}(x) \int_{K} \int_{\widehat{K}}\left|D_{h_{0}^{-1}} \varphi(x k, \omega \xi)\right|^{2} d m_{K}(k) d m_{\widehat{K}}(\omega) \\
& =\|\psi\|_{2}^{2}\|\varphi\|_{2}^{2} .
\end{aligned}
$$

So $\varphi=0$ and $M^{\perp}=\{0\}$.

Theorem 3.2. Let $G_{\wp}:=H \rtimes_{\wp}(K \times \widehat{K} \times \widehat{K} \times K)$ be the generalized wave packet group then the representation $\left(\Lambda, L^{2}(K \times \widehat{K})\right)$ has an admissible vector (and so all vectors are admissible) if and only if $H$ is compact.

Proof. Suppose $\psi \in L^{2}(K \times \widehat{K})$. Applying (1.2) we obtain

$$
\begin{aligned}
C_{\psi}= & \int_{G_{\wp}}|\langle\psi, \Lambda(h, k, \omega, \xi, x) \psi\rangle|^{2} d m_{H}(h) d m_{K}(k) d m_{\widehat{K}}(\xi) d m_{\widehat{K}}(\omega) d m_{K}(x) \\
= & \int_{G_{\wp}}\left|\left\langle T_{\left(k^{-h}, \bar{\omega}_{h}\right)} \psi, M_{\left(\xi_{h}, x^{h}\right)} \mathfrak{D}_{h} \psi\right\rangle\right|^{2} d m_{H}(h) d m_{K}(k) d m_{\widehat{K}}(\xi) d m_{\widehat{K}}(\omega) d m_{K}(x) \\
= & \int_{G_{\wp}}\left|\int_{K} \int_{\widehat{K}} \overline{T_{\left(\bar{\xi}_{h}, x^{-h}\right)} \widehat{\mathfrak{D}_{h}} \widehat{\psi} \cdot \widehat{\psi}}(\gamma, y) \omega_{h}(y) \overline{\gamma\left(k^{-h}\right)} d m_{\widehat{K}}(\gamma) d m_{K}(y)\right|^{2} \\
& d m_{H}(h) d m_{K}(k) d m_{\widehat{K}}(\xi) d m_{\widehat{K}}(\omega) d m_{K}(x) \\
= & \int_{G_{\wp}}\left|\mathcal{F}\left(T_{\left(\bar{\xi}_{h}, \tau_{h}-1(x)\right)} \widehat{\mathfrak{D}_{h}} \widehat{\psi} \cdot \widehat{\psi}\right)\left(k^{h^{-1}}, \overline{\omega_{h}}\right)\right|^{2} d m_{H}(h) d m_{K}(k) d m_{\widehat{K}}(\xi) d m_{\widehat{K}}(\omega) d m_{K}(x)
\end{aligned}
$$




$$
\begin{aligned}
& =\int_{H} \int_{\widehat{K}} \int_{K} \int_{K} \int_{\widehat{K}}\left|\mathcal{F}\left(T_{\left(\bar{\xi}_{h}, \tau_{h^{-1}}(x)\right)} \widehat{\mathfrak{D}_{h}} \widehat{\psi} \cdot \widehat{\psi}\right)\left(k^{-1}, \bar{\omega}\right)\right|^{2} d m_{K}\left(k^{h^{-1}}\right) d m_{\widehat{K}}\left(\omega_{h^{-1}}\right) d m_{H}(h) \\
& d m_{\widehat{K}}(\xi) d m_{K}(x) \\
& =\int_{H} \int_{\widehat{K}} \int_{K} \int_{\widehat{K}} \int_{K}\left|T_{\left(\bar{\xi}_{h}, \tau_{h}^{-1}(x)\right)} \widehat{\mathfrak{D}_{h}} \widehat{\psi}(\gamma, y)\right|^{2}|\widehat{\psi}(\gamma, y)|^{2} d m_{H}(h) d m_{K}(y) d m_{\widehat{K}}(\xi) d m_{\widehat{K}}(\gamma) d m_{K}(x) \\
& =\int_{H} \int_{\widehat{K}} \int_{K} \int_{\widehat{K}} \int_{K}\left|\widehat{\mathfrak{D}_{h}} \widehat{\psi}\left(\gamma \xi_{h}, y \tau_{h}(x)\right)\right|^{2}|\widehat{\psi}(\gamma, y)|^{2} d m_{\widehat{K}}(\gamma) d m_{K}(y) d m_{H}(h) d m_{\widehat{K}}(\xi) d m_{K}(x) \\
& =\int_{H} \int_{\widehat{K}} \int_{K} \int_{\widehat{K}} \int_{K}\left|\widehat{\mathfrak{D}_{h}} \widehat{\psi}(\xi, x)\right|^{2} d m_{\widehat{K}}(\xi) d m_{K}(x)|\widehat{\psi}(\gamma, y)|^{2} d m_{K}(y) d m_{\widehat{K}}(\gamma) d m_{H}(h) \\
& =\int_{\widehat{K}} \int_{K} \int_{H}\left|\widehat{\mathfrak{D}_{h}} \widehat{\psi}(\xi, x)\right|^{2} d m_{H}(h) d m_{K}(x) d m_{\widehat{K}}(\xi) \int_{\widehat{K} \times K}|\widehat{\psi}(\gamma, y)|^{2} d m_{K}(y) d m_{\widehat{K}}(\gamma) \\
& =\|\psi\|_{2}^{4} m_{H}(H) \text {. }
\end{aligned}
$$

Since $m_{H}(H)<\infty$ follows that $H$ is compact, this immediately follows the result.

Corollary 3.3. Let $G_{\wp}$ be the generalized wave packet group and $\psi \in L^{2}(K \times \widehat{K})$ an admissible vector for $\left(\Lambda, L^{2}(K \times \widehat{K})\right)$. Then

(1) The generalized wave packet system $\mathcal{T}(\psi):=\left\{\mathfrak{D}_{h} T_{(k, \omega)} M_{(\xi, x)} \psi ; \quad(h, k, \omega, \xi, x) \in\right.$ $\left.G_{\wp}\right\}$ is a tight representation frame, in particular,

$$
\varphi=\|\psi\|_{2}^{-2} \int_{G_{\wp}}\left\langle\varphi, \mathfrak{D}_{h} T_{(k, \omega)} M_{(\xi, x)} \psi\right\rangle \mathfrak{D}_{h} T_{(k, \omega)} M_{(\xi, x)} \psi d m_{G_{\wp}}(h, k, \omega, \xi, x)
$$

(2) The following orthogonality relation for all $\varphi_{1}, \varphi_{2} \in L^{2}(K \times \widehat{K})$ holds.

$$
\int_{G_{\wp}}\left\langle\varphi_{1}, \Lambda(h, k, \omega, \xi, x) \psi\right\rangle\left\langle\Lambda(h, k, \omega, \xi, x) \psi, \varphi_{2}\right\rangle d m_{G_{\wp}}(h, k, \omega, \xi, x)=C_{\psi}^{1 / 2}\left\langle\varphi_{1}, \varphi_{2}\right\rangle .
$$

Proof. Using Theorem 3.2 follows that

$$
\int_{G_{\wp}}|\langle\varphi, \Lambda(h, k, \omega, \xi, x) \psi\rangle|^{2} d m_{H}(h) d m_{K}(k) d m_{\widehat{K}}(\xi) d m_{\widehat{K}}(\omega) d m_{K}(x)=\|\psi\|_{2}^{2}\|\varphi\|_{2}^{2},
$$

for all $\varphi \in L^{2}(K \times \widehat{K})$. In other words, the family $\mathcal{T}(\psi)$ is a tight representation frame with the bound $\|\psi\|_{2}^{2}$. This proves (1), and (2) follows immediately by (1).

\section{Characterizations of dual representation frames}

In this section, we intend to discuss on the duality of representation frames. Several authors proposed necessary conditions for dual frames $[6,11,12]$. In [8], Chui and Shi give necessary conditions for dual frames on $L^{2}(\mathbb{R})$. The construction of dual on bandlimited framelets with desired time localization can be found in [32].

Let $(X, \nu)$ be a measure space. For two Bessel families $F, G: X \rightarrow \mathcal{H}$ we define the operator $S_{F, G}$ on $\mathcal{H}$ weakly via

$$
S_{F, G} f=\int_{X}\langle f, F(x)\rangle G(x) d \nu(x) .
$$

Bessel families $F$ and $G$ are called dual if and only if $S_{F, G}=I$. Further, suppose $S_{F, G}$ is invertible, then it is easy to see that $S_{F}^{-1} F+S_{F} S_{F, G}^{-1} G-F$ is a dual frame for $F$. We collect in the next proposition some characterizations of continuous dual frames. 
Proposition 4.1. Let $F, G: X \rightarrow \mathcal{H}$ be Bessel families. The following are equivalent:

(1) $F$ and $G$ are continuous dual frames.

(2) $F$ and $S_{F}^{-1} F+S_{F} G-F$ are continuous dual frames.

(3) There exists a Bessel family $L: X \rightarrow \mathcal{H}$ such that $G(x)=S^{-1} F(x)+L(x)$ and

$$
\int_{X} L(x)\langle f, F(x)\rangle d \nu(x)=0 .
$$

The characterization of our dual frames, which introduced in the previous section, are given in the next subsections.

\subsection{Dual of wave packet frames}

In general, the canonical dual frame of a wavelet system has not the wavelet structure [10]. In the sequel, we introduce many duals which have the same structure of the original frames, and therefore, they are suitable for applications.

Theorem 4.2. Let $\psi, \widetilde{\psi} \in L^{2}(K \times \widehat{K})$. The following are equivalent:

(1) $\mathbb{F}(\psi)$ and $\mathbb{F}(\widetilde{\psi})$ are dual representation frames.

(2) $\alpha_{\psi, \widetilde{\psi}}(\xi, y):=\int_{H} \widehat{\mathfrak{D}_{h}} \widehat{\widetilde{\psi}}(\xi, y) \widehat{\mathfrak{D}_{h}} \widehat{\psi}(\xi, y) d m_{H}(h)=1, \quad$ a.e. $\quad(\xi, y) \in \widehat{K} \times K$.

(3) There exists $\varphi \in L^{2}(K \times \widehat{K})$ such that $\widetilde{\psi}:=S_{\psi}^{-1} \psi+\varphi$ and $\operatorname{Im} W_{\varphi} \perp \operatorname{Im} W_{\psi}$.

(4) There exists $\varphi \in L^{2}(K \times \widehat{K})$ such that $\widetilde{\psi}:=S_{\psi}^{-1} \psi+\varphi$ and $\alpha_{\psi, \varphi}=0$.

Proof. Define the operator $S_{\psi, \widetilde{\psi}}$ on $L^{2}(K \times \widehat{K})$ weakly by

$$
S_{\psi, \widetilde{\psi}} \varphi=\int_{G_{\Theta}}\left\langle\varphi, T_{(k, \omega)} \mathfrak{D}_{h} \psi\right\rangle T_{(k, \omega)} \mathfrak{D}_{h} \widetilde{\psi} d m_{H}(h) d m_{K}(k) d m_{\widehat{K}}(\omega) .
$$

Obviously, $\psi$ and $\widetilde{\psi}$ generate wave packet dual representation frames if and only if $S_{\psi, \widetilde{\psi}}$ is the identity operator.

$(2) \Rightarrow(1)$. Using Lemma 2.3 yields

$$
\begin{aligned}
Z_{c} S_{\psi, \widetilde{\psi}} \varphi(\xi, y)= & \int_{G_{\Theta}}\left\langle\varphi, T_{(k, \omega)} \mathfrak{D}_{h} \psi\right\rangle Z_{c} T_{(k, \omega)} \mathfrak{D}_{h} \widetilde{\psi}(\xi, y) d m_{H}(h) d m_{K}(k) d m_{\widehat{K}}(\omega) \\
= & \int_{H}\left(Z_{c} \mathfrak{D}_{h} \widetilde{\psi}\right)(\xi, y) \int_{K \times \widehat{K}} \int_{\widehat{K} \times K}\left(Z_{c} \varphi \overline{Z_{c} \mathfrak{D}_{h} \psi}\right)(\gamma, s) \gamma(k) \\
& \omega(s) \overline{\xi(k) \omega(y)} d m_{H}(h) d m_{\widehat{K}}(\gamma) d m_{K}(s) d m_{\widehat{K}}(\omega) d m_{K}(k) \\
= & \int_{H}\left(Z_{c} \mathfrak{D}_{h} \widetilde{\psi}\right)(\xi, y) Z_{c} \varphi(\xi, y) \overline{\left(Z_{c} \mathfrak{D}_{h} \psi\right)(\xi, y)} d m_{H}(h) \\
= & Z_{c} \varphi(\xi, y) \alpha_{\psi, \widetilde{\psi}}(\xi, y) .
\end{aligned}
$$

Therefore, $\widehat{S_{\psi, \widetilde{\psi}}}=\alpha_{\psi, \widetilde{\psi}} \widehat{\varphi}$, and hence $S_{\psi, \widetilde{\psi}}=I$. Thus, $\psi$ and $\widetilde{\psi}$ generate wave packet dual representation frames. Conversely, let $\mathbb{F}(\psi)$ and $\mathbb{F}(\widetilde{\psi})$ be dual frames. Then $\alpha_{\psi, \widetilde{\psi}} \widehat{f}=\widehat{f}$, for every $f \in L^{2}(K \times \widehat{K})$. Applying Lemma 4.19 of [14] we have $\alpha_{\psi, \widetilde{\psi}}=1$, a.e.. 
$(1) \Leftrightarrow(3)$ Combining (2.2) and (2.5) we obtain

$$
\begin{aligned}
\widehat{\mathfrak{D}}_{h}\left(\widehat{S_{\psi}^{-1}} \psi\right)(\omega, x) & =\left(\frac{1}{\gamma_{\psi}} \widehat{\psi}\right)\left(\widehat{\tau_{h}^{-1}}(\omega), \tau_{h}^{-1}(x)\right) \\
& =\left(\frac{1}{\gamma_{\psi}\left(\widehat{\tau_{h}^{-1}}(\omega), \tau_{h}^{-1}(x)\right)}\right) \widehat{\psi}\left(\widehat{\tau_{h}^{-1}}(\omega), \tau_{h}^{-1}(x)\right) \\
& =\frac{1}{\gamma_{\psi}(\omega, x)} \widehat{\psi}\left(\widehat{\tau_{h}^{-1}}(\omega), \tau_{h}^{-1}(x)\right) \\
& =\widehat{S_{\psi}^{-1}} \widehat{\mathfrak{D}_{h} \psi}(\omega, x) .
\end{aligned}
$$

Hence,

$$
\widehat{T_{(k, \omega)} \mathfrak{D}_{h}} \widehat{S_{\psi}^{-1} \psi}=\widehat{S_{\psi}^{-1}} \widehat{T_{(k, \omega)} \mathfrak{D}} h .
$$

So $S_{\psi}^{-1}$ commutes with the translation and modulation operators. Putting $\varphi=\widetilde{\psi}-S_{\psi}^{-1} \psi$, we have

$$
T_{(k, \omega)} \mathfrak{D}_{h} \widetilde{\psi}=S_{\psi}^{-1} T_{(k, \omega)} \mathfrak{D}_{h} \psi+T_{(k, \omega)} \mathfrak{D}_{h} \varphi
$$

Due to Proposition $4.1(3)$ the families $\mathbb{F}(\psi)$ and $\mathbb{F}(\widetilde{\psi})$ are dual frames if and only if

$$
\left\langle W_{\varphi} \eta, W_{\psi} \widetilde{\eta}\right\rangle=\int_{G_{\Theta}}\left\langle\eta, T_{(k, \omega)} \mathfrak{D}_{h} \varphi\right\rangle\left\langle\widetilde{\eta}, T_{(k, \omega)} \mathfrak{D}_{h} \psi\right\rangle d m_{H}(h) d m_{K}(k) d m_{\widehat{K}}(\omega)=0,
$$

for all $\eta$ and $\widetilde{\eta}$ in $L^{2}(K \times \widehat{K})$.

$(1) \Leftrightarrow(4)$ If $\mathbb{F}(\psi)$ and $\mathbb{F}(\widetilde{\psi})$ are dual representation frames, then $\alpha_{\psi, \widetilde{\psi}}=\int_{H} \widehat{\mathfrak{D}_{h}} \widehat{\widetilde{\psi}}(\xi, y) \widehat{\widehat{\mathfrak{D}_{h}} \widehat{\psi}(\xi, y)} d m_{H}(h)=1$, by $(2)$. Putting $\varphi:=\widetilde{\psi}-S_{\psi}^{-1} \psi$ then $\widetilde{\psi}=$ $S_{\psi}^{-1} \psi+\varphi$. Similarly, $\mathbb{F}(\psi)$ and $\mathbb{F}\left(S^{-1} \psi\right)$ are dual representation frames, it follows that

$$
\int_{H} \widehat{\mathfrak{D}_{h}} \widehat{S^{-1} \psi}(\xi, y) \overline{\widehat{\mathfrak{D}_{h}} \widehat{\psi}(\xi, y)} d m_{H}(h)=1 .
$$

Hence,

$$
\alpha_{\psi, \varphi}=\int_{H} \widehat{\mathfrak{D}_{h}} \widehat{\widetilde{\psi}}(\xi, y) \overline{\widehat{\mathfrak{D}_{h}} \widehat{\psi}(\xi, y)} d m_{H}(h)-\int_{H} \widehat{\mathfrak{D}_{h}} \widehat{S^{-1} \psi}(\xi, y) \overline{\widehat{\mathfrak{D}_{h}} \widehat{\psi}(\xi, y)} d m_{H}(h)=0 .
$$

Conversely, if there exists $\varphi \in L^{2}(K \times \widehat{K})$ such that $\widetilde{\psi}:=S_{\psi}^{-1} \psi+\varphi$ and $\alpha_{\psi, \varphi}=0$, then

$$
\alpha_{\psi, \widetilde{\psi}}=\int_{H} \widehat{\mathfrak{D}_{h}} \widehat{S^{-1} \psi}(\xi, y) \widehat{\widehat{\mathfrak{D}_{h}}} \widehat{\psi}(\xi, y) d m_{H}(h)+\alpha_{\psi, \varphi}=1 .
$$

Thus, $\mathbb{F}(\psi)$ and $\mathbb{F}(\widetilde{\psi})$ are dual representation frames by $(2)$.

Corollary 4.3. Let $G_{\Theta}=H \ltimes_{\Theta}(K \times \widehat{K})$ and $\psi, \widetilde{\psi} \in L^{2}(K \times \widehat{K})$ generate continuous wave packet representation frames. The following are equivalent:

(1) $\mathbb{F}(\psi)$ and $\mathbb{F}(\widetilde{\psi})$ are dual frames.

(2) $\mathbb{F}(\psi)$ and $\mathbb{F}\left(2 \widetilde{\psi}-S_{\psi}^{-1} \psi\right)$ are dual frames.

(3) $\mathbb{F}(\psi)$ and $\mathbb{F}\left(2 S_{\psi}^{-1} \psi-\frac{1}{\alpha_{\psi, \widetilde{\psi}}} \widetilde{\psi}\right)$ are dual frames.

(4) $\mathbb{F}(\psi)$ and $\mathbb{F}\left(S_{\psi}^{-1} \psi+S_{\psi} \widetilde{\psi}-\psi\right)$ are dual frames.

(5) $\mathbb{F}(\psi)$ and $\mathbb{F}\left(S_{\psi}^{-1} \psi-\frac{1}{\gamma_{\psi}} \psi+\widetilde{\psi}\right)$ are dual frames. 
Corollary 4.4. Let $\psi, \widetilde{\psi} \in L^{2}(K \times \widehat{K})$ and $\alpha_{\psi, \widetilde{\psi}} \neq 0$, a.e.. Then $\mathcal{F}(\mathbb{F}(\psi))$ and $\frac{1}{\alpha_{\psi, \widetilde{\psi}}} \mathcal{F}(\mathbb{F}(\widetilde{\psi}))$ are dual frames. In particular,

$$
\widehat{\varphi}=\frac{1}{\alpha_{\psi, \widetilde{\psi}}} \int_{G_{\Theta}}\left\langle\varphi, T_{(k, \omega)} \mathfrak{D}_{h} \psi\right\rangle \mathcal{F}\left(T_{(k, \omega)} \mathfrak{D}_{h} \widetilde{\psi}\right) d m_{H}(h) d m_{K}(k) d m_{\widehat{K}}(\omega) .
$$

\subsection{Dual of generalized wave packet frames}

In this subsection suppose $G_{\wp}:=H \ltimes_{\wp}(K \times \widehat{K} \times \widehat{K} \times K)$, and

$$
\mathcal{T}(\psi):=\left\{\mathfrak{D}_{h} T_{(k, \omega)} M_{(\xi, x)} \psi ; \quad(h, k, \omega, \xi, x) \in G_{\wp}\right\}
$$

is a generalized wave packet representation frame for some $\psi \in L^{2}(K \times \widehat{K})$. In the sequel, we characterize dual of such continuous frames.

Theorem 4.5. Let $\psi, \widetilde{\psi} \in L^{2}(K \times \widehat{K})$ and $H$ be a compact group. The following are equivalent:

(1) $\mathcal{T}(\psi)$ and $\mathcal{T}(\widetilde{\psi})$ are dual represetation frames.

(2) $\langle\psi, \widetilde{\psi}\rangle=1$.

(3) $\widetilde{\psi}:=S_{\psi}^{-1} \psi+\varphi$ for some $\varphi \in L^{2}(K \times \widehat{K})$ such that $\operatorname{Im} W_{\varphi} \perp \operatorname{Im} W_{\psi}$.

(4) There exists $\varphi \in L^{2}(K \times \widehat{K})$ such that $\widetilde{\psi}:=S_{\psi}^{-1} \psi+\varphi$ and $\langle\psi, \varphi\rangle=0$.

Proof. The operator $S_{\psi, \widetilde{\psi}}$ on $L^{2}(K \times \widehat{K})$ is defined weakly by

$\mathcal{S}_{\psi, \widetilde{\psi}} \varphi=\int_{G_{\wp}}\left\langle\varphi, \mathfrak{D}_{h} T_{(k, \omega)} M_{(k, \omega)} \psi\right\rangle \mathfrak{D}_{h} T_{(k, \omega)} M_{(k, \omega)} \psi d m_{H}(h) d m_{K}(k) d m_{\widehat{K}}(\omega) d m_{\widehat{K}}(\xi) d m_{K}(x)$.

$(2) \Leftrightarrow(1)$ A straightforward computation shows that

$$
Z_{c} \mathcal{S}_{\psi, \widetilde{\psi}} \varphi(\gamma, s)=Z_{c} \varphi(\gamma, s) \sigma(\gamma, s)
$$

where

$$
\begin{aligned}
\sigma(\gamma, s) & =\int_{H} \int_{\widehat{K}} \int_{K} Z_{c} \mathfrak{D}_{h} M_{(\xi, x)} \psi(\gamma, s) \overline{Z_{c} \mathfrak{D}_{h} M_{(\xi, x)} \widetilde{\psi}(\gamma, s)} d m_{H}(h) d m_{\widehat{K}}(\xi) d m_{K}(x) \\
& =\int_{H} \int_{\widehat{K}} \int_{K} \widehat{\psi}\left(\gamma_{h^{-1}} \xi, \tau_{h^{-1}}(s) x\right) \overline{\widehat{\widetilde{\psi}}\left(\gamma_{h^{-1}} \xi, \tau_{h^{-1}}(s) x\right)} d m_{H}(h) d m_{\widehat{K}}(\xi) d m_{K}(x) \\
& =m_{H}(H)\langle\psi, \widetilde{\psi}\rangle \\
& =\langle\psi, \widetilde{\psi}\rangle .
\end{aligned}
$$

This follows that

$$
\mathcal{S}_{\psi, \widetilde{\psi}}=\langle\psi, \widetilde{\psi}\rangle I .
$$

Therefore $\mathcal{T}(\psi)$ and $\mathcal{T}(\widetilde{\psi})$ are dual representation frames if and only if $\langle\psi, \widetilde{\psi}\rangle=1$.

By analysis the argument in the proof of Theorem 4.2 we can prove the rest.

The following conclusions can be derived directly from the above theorem.

Corollary 4.6. Let $H$ be a compact group. Then

(1) $\mathcal{T}(\psi)$ and $\frac{1}{\|\psi\|^{2}} \mathcal{T}(\psi)$ are dual representation frame.

(2) If $\|\psi\|_{2}\|\widetilde{\psi}\|_{2}<1$ then $\mathcal{T}(\psi)$ and $\mathcal{T}(\widetilde{\psi})$ can not be dual representation frames.

Corollary 4.7. Let $\psi, \widetilde{\psi} \in L^{2}(K \times \widehat{K})$ and $H$ be a compact group. The following are equivalent:

(1) $\mathcal{T}(\psi)$ and $\mathcal{T}(\widetilde{\psi})$ are dual representation frames.

(2) $\mathcal{T}(\psi)$ and $\mathcal{T}\left(2 \widetilde{\psi}-\frac{1}{\|\psi\|^{2}} \psi\right)$ are dual representation frames. 
(3) $\mathcal{T}(\psi)$ and $\mathcal{T}\left(2 \widetilde{\psi}-S_{\psi}^{-1} \psi\right)$ are dual representation frames.

(4) $\mathcal{T}(\psi)$ and $\mathcal{T}\left(2\|\widetilde{\psi}\|^{2} S_{\psi}^{-1} \widetilde{\psi}-S_{\psi}^{-1} \psi\right)$ are dual representation frames.

(5) $\mathcal{T}(\psi)$ and $\mathcal{T}\left(S_{\psi}^{-1} \psi+S_{\psi}^{-1} \widetilde{\psi}-\psi\right)$ are dual representation frames.

(6) $\mathcal{T}(\psi)$ and $\mathcal{T}(\widetilde{\psi}+\varphi)$, for some $\varphi$ with $\langle\varphi, \psi\rangle=0$ are dual representation frames.

Proof. $(1) \Leftrightarrow(2)$ If $\mathcal{T}(\psi)$ and $\mathcal{T}(\widetilde{\psi})$ are dual representation frames, then $\langle\psi, \widetilde{\psi}\rangle=1$ by Theorem 4.5, so

$$
\begin{aligned}
\left\langle\psi, 2 \widetilde{\psi}-\frac{1}{\|\psi\|^{2}} \psi\right\rangle & =\langle\psi, 2 \widetilde{\psi}\rangle-\left\langle\psi, \frac{1}{\|\psi\|^{2}} \psi\right\rangle \\
& =2\langle\psi, \widetilde{\psi}\rangle-\frac{1}{\|\psi\|^{2}}\langle\psi, \psi\rangle=1
\end{aligned}
$$

Hence, $\mathcal{T}(\psi)$ and $\mathcal{T}\left(2 \widetilde{\psi}-\frac{1}{\|\psi\|^{2}} \psi\right)$ are dual representation frames by Theorem 4.5. Conversely, if $\mathcal{T}(\psi)$ and $\mathcal{T}\left(2 \widetilde{\psi}-\frac{1}{\|\psi\|^{2}} \psi\right)$ are dual representation frames then $\left\langle\psi, 2 \widetilde{\psi}-\frac{1}{\|\psi\|^{2}} \psi\right\rangle=$ 1 and so $\langle\psi, \widetilde{\psi}\rangle=1$, by using Theorem 4.5 it follows that $\mathcal{T}(\psi)$ and $\mathcal{T}(\widetilde{\psi})$ are dual representation frames. The rest follow by a similar argument.

We end this section with a perturbation result on duals.

Theorem 4.8. Let $H$ be a compact group and $\psi, \widetilde{\psi}, \varphi \in L^{2}(K \times \widehat{K})$ such that $\|\psi-\varphi\|_{2}\|\widetilde{\psi}\|_{2}<1$, also let $\mathcal{T}(\psi)$ and $\mathcal{T}(\widetilde{\psi})$ be dual representation frames. Then $\mathcal{T}(\widetilde{\psi})$ and $S_{\varphi, \widetilde{\psi}}^{-1}(\mathcal{T}(\varphi))$ are also dual representation frames.

Proof. For any $\eta \in L^{2}(K \times \widehat{K})$ we obtain

$$
\begin{aligned}
\left\|\left(T_{\mathcal{T}(\psi)}^{*}-T_{\mathcal{T}(\varphi)}^{*}\right) \eta\right\|_{2}^{2} & =\int_{G_{\Theta}}|\langle\eta, \mathcal{T}(\psi)-\mathcal{T}(\varphi)\rangle|^{2} d m_{G_{\Theta}} \\
& =\left\|W_{\psi-\varphi} \eta\right\|_{2}^{2} \\
& =\|\psi-\varphi\|_{2}^{2}\|\eta\|_{2}^{2} .
\end{aligned}
$$

Therefore,

$$
\begin{aligned}
\left\|I-S_{\varphi, \widetilde{\psi}}\right\| & =\left\|T_{\mathcal{T}(\widetilde{\psi})}\left(T_{\mathcal{T}(\psi)}^{*}-T_{\mathcal{T}(\varphi)}^{*}\right)\right\| \\
& \leq\|\widetilde{\psi}\|_{2}\|\psi-\varphi\|_{2}<1 .
\end{aligned}
$$

So $S_{\varphi, \widetilde{\psi}}$ is invertible. This easily follows that $\mathcal{T}(\widetilde{\psi})$ and $S_{\varphi, \widetilde{\psi}}^{-1}(\mathcal{T}(\varphi))$ are dual pairs.

\section{Examples and conclusions}

Throughout this section, we present some examples to illustrate our results. More precisely, we discuss on the existence of admissible vectors of wave packet and generalized wave packet groups. See the basic properties of the following examples in [19,20].

Example 5.1. Consider the wave packet group $G_{\Theta}=\mathbb{R}^{+} \ltimes_{\Theta}(\mathbb{R} \times \mathbb{R})$ where $\Theta: \mathbb{R}^{+} \rightarrow$ $\operatorname{Aut}(\mathbb{R} \times \mathbb{R})$ is given on $L^{2}(K \times \widehat{K})$ by $a \mapsto \Theta_{a}(x, \omega)=\left(a x, a^{-1} \omega\right)$. The dilation operator is defined by $\mathcal{D}_{a} \varphi(k, \omega)=\varphi\left(a^{-1} k, a \omega\right)$ and

$$
U(a, k, \omega) \varphi(x, \xi)=\varphi\left(a^{-1} x k^{-1}, a \xi \bar{\omega}\right)
$$


is denoted the quasi regular representation on $G_{\Theta}$. Using Theorem 2.4 follows that $\left\{T_{(k a, \omega a)} \psi\right\}_{(a, k, \omega) \in G_{\Theta}}$ is a wave packet representation frame if and only if

$$
A \leq \int_{\mathbb{R}^{+}}\left|\widehat{\psi}\left(a \xi, a^{-1} x\right)\right|^{2} \frac{d a}{|a|} \leq B, \quad \text { a.e. }(\xi, x) \in \mathbb{R}^{2} .
$$

For example, suppose $\psi_{1} \in L^{2}(\mathbb{R})$ is an admissible vector for the affine group $\mathbb{R}^{+} \ltimes_{\tau} \mathbb{R}$, then $\Psi \in L^{2}\left(\mathbb{R}^{2}\right)$ defined by

$$
|\widehat{\Psi}|=\left|\widehat{\psi}_{1}\right|^{1 / 2} \otimes\left|\widehat{\psi}_{1}\right|^{1 / 2}
$$

is an admissible vector for $\left(U, L^{2}\left(\mathbb{R}^{2}\right)\right)$. In particular, if $\psi_{1} \in L^{2}(\mathbb{R})$ whose Fourier transform satisfies

$$
\widehat{\psi_{1}}=\chi_{[\pi, 2 \pi]}
$$

and $\Psi$ is defined by $\widehat{\Psi}=\widehat{\psi}_{1}^{1 / 2} \otimes \widehat{\psi}_{1}^{1 / 2}$, then

$$
\begin{aligned}
\int_{\mathbb{R}^{+}}\left|\widehat{\Psi}\left(a \xi, a^{-1} x\right)\right|^{2} \frac{d a}{|a|} & \leq\left(\int_{\mathbb{R}^{+}}\left|\widehat{\psi}_{1}(a \xi)\right|^{2} \frac{d a}{|a|}\right)^{1 / 2}\left(\int_{\mathbb{R}^{+}}\left|\widehat{\psi}_{1}\left(a^{-1} x\right)\right|^{2} \frac{d a}{|a|}\right)^{1 / 2} \\
& =\int_{\mathbb{R}^{+}}\left|\widehat{\psi}_{1}(a)\right|^{2} \frac{d a}{|a|}=\int_{\pi}^{2 \pi} \frac{d a}{|a|}=\operatorname{Ln} 2 .
\end{aligned}
$$

Also for vectors $\varphi$ and $\psi$ in $L^{2}\left(\mathbb{R}^{2}\right)$ such that

$$
\int_{\mathbb{R}^{+}} \overline{\widehat{\psi}\left(a \xi, a^{-1} x\right)} \hat{\varphi}\left(a \xi, a^{-1} x\right) \frac{d a}{|a|}=1, \quad \text { a.e. }(\xi, x) \in \mathbb{R}^{2},
$$

The families $\left\{T_{(k a, \omega a)} \varphi\right\}_{(a, k, \omega) \in G_{\Theta}}$ and $\left\{T_{(k a, \omega a)} \psi\right\}_{(a, k, \omega) \in G_{\Theta}}$ are dual representation frames.

Example 5.2. The generalized wave packet group $G_{\wp}=\{1,2, \ldots, p-1\} \ltimes_{\wp} \mathbb{Z}_{p} \times \mathbb{Z}_{p} \times \mathbb{Z}_{p} \times \mathbb{Z}_{p}$, where $m_{p}(s)=p s$ and $\wp_{m}(s, \omega, \xi, x)=\left(m s, m_{p} \omega, m_{p} \xi, m x\right)$, is a finite non-abelian group. The dilation operator is given by $\mathfrak{D}_{m} \varphi(s, \hat{s})=\varphi\left(m_{p} s, m \dot{s}\right)$ for $\varphi \in \mathbb{C}^{p \times p}$. Using Theorem 3.2 follows that every $\psi \in \mathbb{C}^{p \times p}$ is an admissible vector and $\left\{\mathfrak{D}_{m} T_{(k, \omega)} M_{(\xi, x)} \psi\right\}$ is a tight representation frame. Also we achieve the following reconstruction

$$
\varphi=\frac{1}{\|\varphi\|_{2}^{2}} \sum_{m=1}^{p-1} \sum_{k=0}^{p-1} \sum_{\omega=0}^{p-1} \sum_{\xi=0}^{p-1} \sum_{x=0}^{p-1}\left\langle\varphi, \mathfrak{D}_{m} T_{(k, \omega)} M_{(\xi, x)} \psi\right\rangle \mathfrak{D}_{m} T_{(k, \omega)} M_{(\xi, x)} \psi, \quad\left(\varphi \in \mathbb{C}^{p \times p}\right) .
$$

Example 5.3. Let $n>1$ be an integer, $H=S O(n)$ and $K=\mathbb{R}^{n}$. We can define the continuous homomorphism $\Theta$ by $A \mapsto \Theta_{A}(x, \omega)=\left(A x, A^{-1} \omega\right)$ for all $A \in S O(n)$ and $(x, \omega) \in \mathbb{R}^{n} \times \mathbb{R}^{n}$. The quasi regular representation $U$ on $G_{\Theta}=S O(n) \ltimes_{\Theta} \mathbb{R}^{n} \times \mathbb{R}^{n}$ is given by $U(A, x, \omega)=T_{(x, \omega)} D_{A}$ that $D_{A} f(x, \omega)=f\left(A^{-1} x, A \omega\right)$. Using Theorem 2.4 the family $\left\{T_{\left(A k, A^{-1} \omega\right)} \psi ; \quad(A, x, \omega) \in G_{\Theta}\right\}$ is a wave packet representation frame with bounds $A$ and $B$ if and only if

$$
A \leq \gamma_{\psi}(\omega, x)=\int_{S O(n)}\left|\widehat{\psi}\left(A \omega, A^{-1} x\right)\right|^{2} d A \leq B, \quad \text { a.e. }(\omega, x) \in \mathbb{R}^{n} \times \mathbb{R}^{n} .
$$

So, if $\widehat{\psi}$ is bounded and compact support, then $\psi$ is an admissible vector for $L^{2}\left(\mathbb{R}^{n} \times \mathbb{R}^{n}\right)$. It guarantees the following reconstruction formula in $L^{2}\left(\mathbb{R}^{n} \times \mathbb{R}^{n}\right)$,

$$
f=\int_{G_{\Theta}}\left\langle\widehat{f}, \gamma_{\psi}^{-1} M_{(k, \omega)} \widehat{D_{A}} \widehat{\psi}\right\rangle T_{\left(A k, A^{-1} \omega\right)} \psi d A d \omega d x .
$$

Example 5.4. Consider the shearlet group, denoted by $\mathbb{S}$, as the semidirect product

$$
\left(\mathbb{R}^{+} \ltimes_{\tau} \mathbb{R}\right) \ltimes_{\Theta} \mathbb{R}^{4}
$$

equipped with the group multiplication given by

$$
(a, s, x, \xi) \cdot(\dot{a}, \dot{s}, \dot{x}, \dot{\xi})=\left(a \dot{a}, s+\dot{s} \sqrt{a},(x, \xi)+\left(S_{s} A_{a} \dot{x}, A_{a}^{-1} S_{s}^{-1} \dot{\xi}\right)\right)
$$


that the shearing matrix $S_{s}$ and the parabolic scaling matrix $A_{a}$ are given by

$$
S_{s}=\left[\begin{array}{ll}
1 & s \\
0 & 1
\end{array}\right], \quad A_{a}=\left[\begin{array}{cc}
a & 0 \\
0 & a^{1 / 2}
\end{array}\right] .
$$

The left Haar measure of $\mathbb{S}$ is $\frac{d a}{a^{3}} d s d t$. Let $M_{a s}=S_{s} A_{a}$, the dilation operator can be writen as

$$
\mathcal{D}_{(a, s)} \psi(x, \xi)=\psi\left(M_{\frac{1}{a}(-s)} x, M_{a s} \xi\right) \quad x, \xi \in \mathbb{R}^{2} .
$$

For $\psi \in L^{2}\left(\mathbb{R}^{4}\right)$ the unitary representation $\Gamma: \mathbb{S} \rightarrow \mathcal{U}\left(L^{2}\left(\mathbb{R}^{4}\right)\right)$ can be defined by

$$
\Gamma(a, s, x, \xi) \psi=T_{(x, \xi)} D_{M_{a s}} \psi \text {. }
$$

Using Theorem 2.4, the shearlet system $S H(\psi)=\left\{T_{(t, \xi)} D_{M_{a s}} \psi ; \quad(a, s, t, \xi) \in \mathbb{S}\right\}$ is a wave packet representation frame if and only if

$$
A \leq \int_{\mathbb{R}^{+}} \int_{\mathbb{R}}\left|\widehat{\psi}\left(M_{a s} x, M_{\frac{1}{a}(-s)} \xi\right)\right|^{2} \frac{d a}{a^{3 / 2}} d s \leq B, \quad\left((x, \xi) \in \mathbb{R}^{4}\right) .
$$

Also $\left\{T_{(t, \xi)} D_{M_{a s}} \psi\right\}_{(a, s, t, \xi) \in S}$ and $\left\{T_{(t, \xi)} D_{M_{a s}} \widetilde{\psi}\right\}_{(a, s, t, \xi) \in S}$ are dual frames if and only if

$$
\alpha_{\psi, \widetilde{\psi}}=\int_{\mathbb{R}^{+}} \int_{\mathbb{R}} \widehat{D}_{M_{a s}} \widehat{\widetilde{\psi}}(\xi, y){\widehat{\widehat{D}_{M}}}_{M_{a s}} \widehat{\psi}(\xi, y) \frac{d a}{a^{3 / 2}} d s=1 .
$$

Notice that the generalized shearlet representation $\Lambda(a, s, k, \omega, \xi, x)=D_{M_{a s}} T_{(t, \xi)} M_{(\xi, x)} \psi$ on generalized shearlet group $G=\mathbb{R}^{+} \times \mathbb{R} \ltimes \mathbb{R}^{8}$ has not any admissible vector by Theorem 3.2 .

Acknowledgment. The authors are grateful to the referees for carefully reading and useful comments.

\section{References}

[1] S.T. Ali, J.P. Antoine and J.P. Gazeau, Coherent States, Wavelets and Their Generalizations, Springer-Verlag, New York, 2000.

[2] F. Andersson, M. Carlsson and L. Tenorio, On the representation of functions with Gaussian wave packets, J. Fourier Anal. Appl. 18, 146-181, 2012.

[3] A. Arefijamaal, The continuous Zak transform and generalized Gabor frames, Mediterr. J. Math. Phys. 10 (1), 353-365, 2013.

[4] A. Arefijamaal and A. Ghaani Farashahi, Zak transform for semidirect product of locally compact groups, Anal. Math. Phys. 3 (3), 263-276, 2013.

[5] A. Arefijamaal and R.A. Kamyabi-Gol, On the square integrability of quasi regular representation on semidirect product groups, J. Geom Anal. 19 (3), 541-552, 2009.

[6] O. Christensen, Pairs of dual Gabor frame generators with compact support and desired frequency localization, Appl. Comput. Harmon. Anal. 20 (3), 403-410, 2006.

[7] O. Christensen, Frames and Bases: An Introductory Course, Birkhäuser, Boston, 2008.

[8] C.K. Chui and X. Shi, Orthonormal wavelets and tight frames with arbitrary real dilation, Appl. Comput. Harmon. Anal. 9 (3), 243-264, 2000.

[9] A. Cordoba and C. Fefferman, Wave packets and Fourier integral operators, Comm. Part. Diff. Equat. 3 (11), 979-1005, 1978.

[10] I. Daubechies, The wavelet transform, time frequency locallization and signal analysis, IEEE Trans. Inform. Theory. 36 (5), 961-1005, 1990.

[11] I. Daubechies and B. Han, The canonical dual frame of a wavelet frame, Harmon. Anal. 12, 269-285, 2002.

[12] I. Daubechies and B. Han, Pairs of dual wavelet frames from any two refinable functions, Constr. Approx. 20, 325-352, 2004. 
[13] J. Epperson, Hermite and Laguerre wave packet expansions, Studia Math. 126 (3), 199-217, 1998.

[14] G.B. Folland, A Course in Abstract Harmonic Analysis, CRCPress, Boca Raton, 1995.

[15] I.M. Gelfand, Eigen function expansions for equations with periodic coefficients, Dokl. Akad. Nauk. SSR 73, 1117-1120, 1950.

[16] A. Ghaani Farashahi, Generalized Weyl-Heisenberg groups, Anal. Math. Phys. 4 (3), 187-197, 2014.

[17] A. Ghaani Farashahi, Abstract harmonic analysis of wave packet transforms over locally compact abelian groups, Anal. Math. Banach. J. 11, 50-71, 2017.

[18] A. Ghaani Farashahi, Square-integrability of metaplectic wave packet representation on $L^{2}(\mathbb{R})$, J. Math. Anal. Appl. 449, 769-92, 2017.

[19] A. Ghaani Farashahi, Theoretical frame properties of wave-packet matrices over prime fields, Linear Multilinear Algebra 11, 2017.

[20] A. Ghaani Farashahi, Square-integrability of multivariate metaplectic wave-packet representations, J. Phys. A 50, 115-202, 2017.

[21] A. Ghaani Farashahi, Multivariate wave-packet transforms, Z. Anal. Anwend. 36 (4), 481-500, 2017.

[22] A. Ghaani Farashahi, Abstract coherent state transforms over homogeneous spaces of compact groups, Complex Anal. Oper. Theory 12, 15-37, 2018.

[23] K. Gröchenig, Aspects of Gabor analysis on locally compact Abelian groups, in: Gabor Analysis and Algorithms, Birkhäuser Boston, 211-231, 1998.

[24] K. Gröchenig, Foundations of Time-Frequency Analysis, Birkhäuser, Boston, 2001.

[25] E. Hernandez, D. Labate and G. Weiss, A unified characterization of reproducing systems generated by a finite family II, J. Geom. Anal. 12 (4), 615-662, 2002.

[26] E. Hewitt and K.A. Ross, Abstract Harmonic Analysis, Springer-Verlag, Berlin, Vol I, 1963.

[27] A.J.E.M. Janssen, The Zak transform: a Signal transform for sampled timecontinuous signals Philips J. Res. 43, 23-69, 1988.

[28] E. Kaniuth and G. Kutyniok, Zeros of the Zak transforms on locally compact abelian groups, Proc. Amer. Math. Soc. 126, 3561-3569, 1998.

[29] T.H. Koornwinder, Wavelets: An Elementary Treatment of Theory and Applications, World Scientific, Singapore, (1993).

[30] G. Kutyniok, A qualitative uncertainty principle for functions generating a Gabor frame on LCA groups, J. Math. Anal. Appl. 279, 580-596, 2003.

[31] D. Labate, G. Weiss and E. Wilson, An approach to the study of wave packet systems, wavelet, frames and operator theory, Contemporary Mathematics 345, 215-235, 2004.

[32] J. Lemvig, Constructing pairs of dual bandlimited framelets with desired time localization, Adv. Comput Math. 30, 231-247, 2009.

[33] V. Runde, Lectures on Amenability, Springer, Berlin, 2002.

[34] J. Zak, Finite translations in solid state physics, Phys. Rev. lett. 19, 1967. 